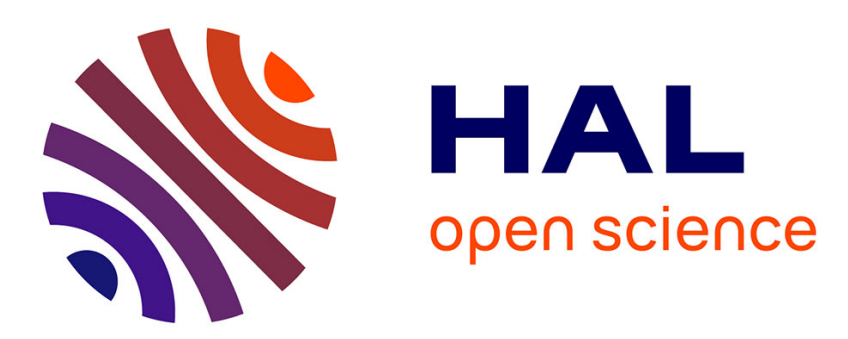

\title{
Structures singulières non linéaires pour les fluides non collisionnels de l'espace des phases
}

\author{
J.R. Burgan, J. Gutierrez, E. Fijalkow, M. Navet, M.R. Feix
}

\section{To cite this version:}

J.R. Burgan, J. Gutierrez, E. Fijalkow, M. Navet, M.R. Feix. Structures singulières non linéaires pour les fluides non collisionnels de l'espace des phases. Journal de Physique Lettres, 1977, 38 (7), pp.161-165. 10.1051/jphyslet:01977003807016100 . jpa-00231350

\section{HAL Id: jpa-00231350 https://hal.science/jpa-00231350}

Submitted on 1 Jan 1977

HAL is a multi-disciplinary open access archive for the deposit and dissemination of scientific research documents, whether they are published or not. The documents may come from teaching and research institutions in France or abroad, or from public or private research centers.
L'archive ouverte pluridisciplinaire HAL, est destinée au dépôt et à la diffusion de documents scientifiques de niveau recherche, publiés ou non, émanant des établissements d'enseignement et de recherche français ou étrangers, des laboratoires publics ou privés. 
Classification

Physics Abstracts

$6.510-6.512-6.540$

\title{
STRUCTURES SINGULIÈRES NON LINÉAIRES POUR LES FLUIDES NON COLLISIONNELS DE L'ESPACE DES PHASES (*)
}

\author{
J. R. BURGAN, J. GUTIERREZ (**), E. FIJALKOW, M. NAVET et M. R. FEIX \\ C.R.P.E.-C.N.R.S. Université d'Orléans, La Source, 45045 Orléans Cedex, France
}

(Reçu le 6 janvier 1977, accepté le 28 février 1977)

\begin{abstract}
Résumé. - On étudie les solutions du système unidimensionnel de Vlasov-Poisson à la fois pour une espèce répulsive (faisceau) et pour un plasma d'électrons en présence d'un fond neutralisant immobile et continu d'ions. Un ansatz sur la structure de la fonction de distribution introduit une solution ayant la forme d'un bâton dans l'espace des phases - solution déjà obtenue dans le cas du faisceau à l'aide d'arguments de self-similarité. Ici nous pouvons généraliser les conditions initiales du bâton et traiter à la fois des problèmes de faisceau et de plasma. Le concept de contamination permet de limiter le bâton et d'éliminer les conditions aux limites non physiques, mais introduit une limitation sur le temps de validité de la solution pour une partie (ou la totalité) des particules. Tant que les particules ne sont pas contaminées un invariant exact peut être obtenu. Des simulations numériques illustrent très bien cette théorie.
\end{abstract}

\begin{abstract}
We study the solutions of the Vlasov-Poisson one dimensional system both for a single repulsive species (beam) and for an electron plasma in presence of a motionless ionic background. An ansatz on the form of the distribution function leads to a solution with a rod structure in phase space. This was already obtained by self-similarity arguments in the beam case. Here we can generalize the initial conditions of the rod and treat both beam and plasma problems. The introduction of the concept of contamination enables the rod to be cut to get rid of non physical boundary conditions but it introduces a time limitation on the validity of the solution for some (or all) particles. As long as particles are not contaminated an invariant can be obtained. Computer simulations support this theory very well.
\end{abstract}

1. Introduction. - Les fluides non collisionnels de l'espace des phases (c'est-à-dire les fluides décrits par les équations de Vlasov et de Poisson) sont au nombre de trois : le plasma où les deux espèces de particules ions et électrons se neutralisent plus ou moins totalement, le gaz d'électrons (ou d'ions) avec une seule espèce de particules se repoussant, et le $g a z$ d'étoiles où l'on retrouve une seule espèce mais avec cette fois des forces attractives.

$\mathrm{Si}$ les solutions linéarisées autour d'un équilibre homogène, qui d'ailleurs n'a de sens que pour le premier des trois fluides, est un problème bien résolu (y compris le traitement par la théorie quasi linéaire d'ondes légèrement amplifiées) les études de solutions fortement non linéaires et dépendantes du temps sont très peu nombreuses. Pratiquement seul le plasma froid a pu être traité [1]. Notre but est de présenter ici quelques autres solutions exactes qui pourraient jouer un rôle important dans l'étude du problème

(*) Communication présentée au Congrès National de Physique des Plasmas, Paris, 6-10 décembre 1976.

(**) Financé par le G.I.F.T. (Espagne). général. Nous verrons que nous pouvons rattacher ces solutions aux solutions self-similaires obtenues par ailleurs [2] et nous déduirons pour certaines particules d'un état initial donné, un invariant exact du mouvement, lequel, suivant le problème, pourra être valable pour un temps infini ou pour un temps fini.

2. Une solution singulière : le bâton infini. Considérons une fonction $\psi$ ayant les propriétés d'une densité de probabilité c'est-à-dire positive et normalisée $\int \psi(x) \mathrm{d} x=1$. Enfin pour simplifier l'interprétation physique admettons que $\psi$ est une fonction paire en $x$. Cherchons une solution de l'équation de Vlasov unidimensionnelle telle que

$$
f(x, v, t)=\psi\left[\frac{v-u(x, t)}{\Phi(t)}\right]
$$

de (1) on déduit aisément la densité $n(x, t)=\Phi(t)$ et la vitesse moyenne au point $x,\langle v\rangle=u(x, t)$. 
Enfin en portant (1) dans l'équation de Vlasov on obtient

$$
\begin{aligned}
& v\left\{\frac{\mathrm{d} \Phi}{\mathrm{d} t}+\Phi(t) \frac{\delta u}{\delta x}\right\}+ \\
& +\Phi(t) \frac{\delta u}{\delta t}-\Phi(t) \frac{e}{m} E(x, t)-u \frac{\mathrm{d} \Phi}{\mathrm{d} t}=0 .
\end{aligned}
$$

(2) doit être identiquement nul quel que soit $v$ ce qui implique d'abord que le terme entre \{ $\}$ de (2) doit être nul. On déduit en utilisant en plus

$$
\begin{aligned}
& J+\varepsilon_{0} \frac{\delta E}{\delta t}=0 \\
& u=-\frac{\dot{\Phi}}{\Phi} x \\
& E=\frac{e}{\varepsilon_{0}} x(\Phi-N)
\end{aligned}
$$

$N$ est la densité des ions (qui forment un fond continu immobile). En portant (3) dans le $2^{\mathrm{e}}$ terme de (2) il vient

$$
\frac{\mathrm{d}}{\mathrm{d} t}\left(\frac{\dot{\Phi}}{\Phi}\right)+\frac{e^{2}}{m \varepsilon_{0}}(\Phi-N)-\frac{\dot{\Phi}^{2}}{\Phi^{2}}=0
$$

qui donne finalement l'équation extrêmement simple

$$
\frac{\mathrm{d}^{2}}{\mathrm{~d} t^{2}}\left(\frac{1}{\Phi}\right)+\Omega_{\mathrm{p}}^{2}\left(\frac{1}{\Phi}\right)=\frac{e^{2}}{m \varepsilon_{0}}
$$

où l'on a posé

$$
\Omega_{\mathrm{p}}^{2}=N e^{2} / m \varepsilon_{0} .
$$

L'équation correspondante pour un faisceau (1 seule espèce répulsive) s'écrit

$$
\frac{\mathrm{d}^{2}}{\mathrm{~d} t^{2}}\left(\frac{1}{\Phi}\right)^{\prime}=\frac{e^{2}}{m \varepsilon_{0}}
$$

Les solutions ainsi obtenues décrivent des structures infinies où la densité $f(x, v, t)$ est constante le long des droites dans l'espace des phases. En conséquence le champ est linéaire en $x$ et la densité indépendante de $x$. Ces droites forment une sorte de bâton dans l'espace des phases d'extension infinie avec des courants qui augmentent indéfiniment aux deux extrémités. Bien entendu il est nécessaire de limiter les solutions pour $x$ grand ne serait-ce que pour n'avoir à considérer une population finie de particules et de couper les deux extrémités du bâton (voir $\S 4)$.

3. Relations avec les solutions self-similaires. Considérons le cas d'un faisceau (une seule espèce de particules) : la solution de (6) est

$$
\frac{1}{\Phi}=\frac{e^{2}}{m \varepsilon_{0}} \frac{t^{2}}{2}+\beta t+\frac{1}{n_{0}}
$$

On a tenu compte du fait que pour $t=0$, $n=\Phi=n_{0}$.

Introduisons comme en [2] la fréquence plasma associée à la densité $n_{0} / 2$ : soit $\Omega^{2}$ son carré, on a alors $n_{0} / n=\Omega^{2} t^{2}+\beta n_{0} t+1$.

$\mathrm{Si}$ on choisit $\beta n_{0}=2 \Omega$ on obtient

$$
\frac{n}{n_{0}}=\frac{1}{(\Omega t+1)^{2}}
$$

(8) n'est autre que la relation obtenue en (2) pour les solutions self-similaires correspondant à $\alpha=-1$ (formules (1) à (6) de [2]). Nous remarquons d'ailleurs que la self-similarité nous obligeait à avoir $\beta=2 \Omega / n_{0}$ tandis que nous pouvons pour (7) prendre $\beta$ quelconque. En particulier, alors que pour les solutions self-similaires nous ne disposons que d'un paramètre qui fixe à la fois la pente et l'écartement du bâton, on dispose ici de deux paramètres qui permettent un choix plus large. Nous étudierons spécialement le cas d'une distribution où $u(x, t=0)=0$, ce qui correspond à prendre $\beta=0$ avec comme solution pour la densité et le champ électrique

$$
n=\frac{n_{0}}{1+\Omega^{2} t^{2}}, \quad \frac{e E}{m}=\frac{2 \Omega^{2} x}{1+\Omega^{2} t^{2}} .
$$

Il faut se rappeler que le cas du plasma (avec le fond continu d'ions) n'avait pu être traité à l'aide des groupes self-similaires. Ici par contre (5) est très facile à résoudre et donne

$$
\frac{1}{n}=\frac{1}{N}\left(1+\alpha \cos \Omega_{\mathrm{p}} t+\beta \sin \Omega_{\mathrm{p}} t\right) .
$$

Si la population d'électrons est telle que

$$
u(x, t=0)=0,
$$

$\beta$ est nul et en introduisant le résultat précédent dans l'équation (3)

$$
\begin{aligned}
& \frac{\Phi}{N}=\frac{n}{N}=\frac{1}{1+\alpha \cos \Omega_{\mathrm{p}} t}, \\
& \frac{e}{m} E=\frac{-\alpha \Omega_{\mathrm{p}}^{2} \cos \Omega_{\mathrm{p}} t}{1+\alpha \cos \Omega_{\mathrm{p}} t} x .
\end{aligned}
$$

4. Coupure du bâton et concept de contamination. Les conditions initiales dans l'espace des phases décrites par (7) et (10) sont caractérisées par des bâtons horizontaux infinis. Nous aimerions, bien entendu, couper à partir d'une certaine distance le bâton de manière à traiter le cas d'une population finie en expansion sous les effets combinés de la charge d'espace et du mouvement balistique et, dans le $2^{\mathrm{e}}$ cas, retenue, par une force proportionnelle à la distance (créee par les ions immobiles).

Considérons alors la situation suivante : à l'instant initial la population est limitée au segment $|-L,+L|$; ces particules seront dénommées réelles. 
Nous complétons alors le bâton, le rendant ainsi infini par l'adjonction d'une population virtuelle. Nous désirons suivre l'évolution de la population réelle sous l'effet de son propre champ. Une première étape consiste à remarquer que nous connaissons l'évolution de la population réelle sous l'effet de la charge d'espace de deux populations. Il suffit pour cela de calculer l'évolution des particules sur les frontières $x= \pm L$ sous l'effet des champs donnés par (9) et (10). Or en admettant une symétrie par rapport à $x=0$, le champ total créé par les particules telles que $|x|>x_{0}$ est nul pour $|x|<x_{0}$. A l'instant initial donc les particules virtuelles ne créent pas de champs pour les particules réelles. Bien sûr du fait de la dispersion thermique, cette position extérieure des particules virtuelles ne subsistera pas toujours et une certaine contamination due à des croisements entre particules réelles et virtuelles se développera. Il faut donc à chaque instant déterminer la zone ou aucune particule virtuelle n'a pu pénétrer. C'est la zone non contaminée où le champ créé par les particules virtuelles est nul. Cette zone non contaminée peut se rétrécir jusqu'à disparaître totalement. Elle peut également se stabiliser, un certain pourcentage de particules échappant à toute contamination, pour lesquelles nous pourrons calculer des invariants exacts.

Ces concepts déjà introduits dans [2] pour le problème du bâton self-similaire avaient porté sur un modèle Water Bag [3]. En fait la solution est plus générale à condition toutefois d'admettre qu'il existe une vitesse $V_{0}$ telle que le pourcentage de particules dont la dispersion $|v-u(x, t)|$ est supérieure à $V_{0}$ est négligeable. Si l'on tient à considérer toutes les vitesses de dispersion possibles et en particulier des vitesses arbitrairement grandes, il est certain que les particules virtuelles présentant un $|v-u|$ très grand et négatif situées au-delà de $x=L$ vont instantanément contaminer tout le système. Or physiquement il est évident qu'un très petit nombre de particules ne va pas radicalement changer le champ total et qu'en conséquence il est parfaitement possible et raisonnable de considérer qu'au-delà de $V_{0}$ (pris égal à 4 ou 5 vitesses thermiques par exemple)। il n'y a plus de particules.

Maintenant, pour résoudre le problème du faisceau il nous faudra tout d'abord calculer l'évolution de la frontière $x=L$. Une particule de cette frontière aura son mouvement régi par

$$
\frac{\mathrm{d}^{2} x}{\mathrm{~d} t^{2}}=\frac{e}{m} E=\frac{2 \Omega^{2} x}{1+\Omega^{2} t^{2}} .
$$

Or la particule telle que $x=L \dot{x}=-V_{0}$ à l'instant initial est celle qui va constamment déterminer la frontière entre les zones non contaminées et contaminées. La solution de (11) pour cette particule est

$$
X_{\mathrm{c}}=\left\{L-\frac{V_{0}}{2 \Omega}\left(\frac{\tau}{1+\tau^{2}}+\operatorname{Arctg} \tau\right)\right\}\left(1+\tau^{2}\right)
$$

avec $\tau=\Omega t$.

Pour $|x|<X_{\mathrm{c}}$ les particules ne sont pas contaminées. Pour des temps très grands $X_{\mathrm{c}}$ croît comme

$$
L\left(1+\tau^{2}\right)\left(1-\frac{V_{0}}{\Omega L} \frac{\Pi}{4}\right)
$$

et le pourcentage de particules non contaminées est $1-(\Pi / 4)\left(V_{0} / L \Omega\right)$. Il dépend bien sûr des dimensions initiales du bâton et plus précisément du rapport $V_{0} / L \Omega$, c'est-à-dire en gros de sa longueur par rapport à la distance de Debye (puisque $V_{0}$ est de l'ordre de $V_{\mathrm{t}}$ et $\Omega=\Omega_{\mathrm{p}} / \sqrt{2}$ ). Enfin une particule avec la position $x$ et la vitesse $v$ tant qu'elle n'est pas rentrée dans la zone contaminée possède un invariant qui est

$$
\bar{v}=\left(v-\frac{2 \tau x \Omega}{1+\tau^{2}}\right)\left(1+\tau^{2}\right) .
$$

L'invariance de $v$ se démontre aisément en remarquant que la solution de (11) muni des conditions initiales $x=\xi \dot{x}=\eta$ est

$$
x=\left\{\xi+\frac{\eta}{2 \Omega}\left(\frac{\tau}{1+\tau^{2}}+\operatorname{Arctg} \tau\right)\right\}\left(1+\tau^{2}\right) .
$$

En calculant $v=\mathrm{d} x / \mathrm{d} t$ à partir de (14) et en rapportant dans (13), il est aisé de montrer que $\bar{v}=\eta$.

Il est alors intéressant d'introduire l'espace des phases modifié $\bar{x}$ et $\bar{v}$ avec $\bar{v}$ donné par (13) et $\bar{x}$ par

$$
\bar{x}=x\left(1+\tau^{2}\right)^{-1} \text {. }
$$

On remarquera que $\mathrm{d} x \mathrm{~d} v=\mathrm{d} \bar{x} \mathrm{~d} \bar{v}$.

Les figures. 1 et 2 illustrent les concepts d'invariant et de zone non contaminée. Elles s'appliquent à une situation initiale Water Bag avec donc une coupure $V_{0}$ bien déterminée. A $t=0$ les plans de phase $x v$ et $\overline{x v}$ coïncident. Au fur et à mesure que le temps s'écoule on voit la zone contaminée s'étendre jusqu'à la valeur limite prévue par (12) $\bar{x}_{1}=L-\left(V_{0} / \Omega\right)(\Pi / 4)$. Ce comportement est indiqué figure 1 tandis que la figure 2 illustre l'invariance de $\bar{v}$ pour les particules qui ne sortent pas de la zone non contaminée.

Enfin nous donnons pour le cas plasma la solution du calcul du mouvement d'une particule de position et de vitesse initiale $\xi$ et $\eta$. Il faut résoudre l'équation différentielle

$$
\frac{\mathrm{d}^{2} x}{\mathrm{~d} t^{2}}=\frac{-\alpha \Omega_{\mathrm{p}}^{2} \cos \Omega_{\mathrm{p}} t}{1+\alpha \cos \Omega_{\mathrm{p}} t} x
$$




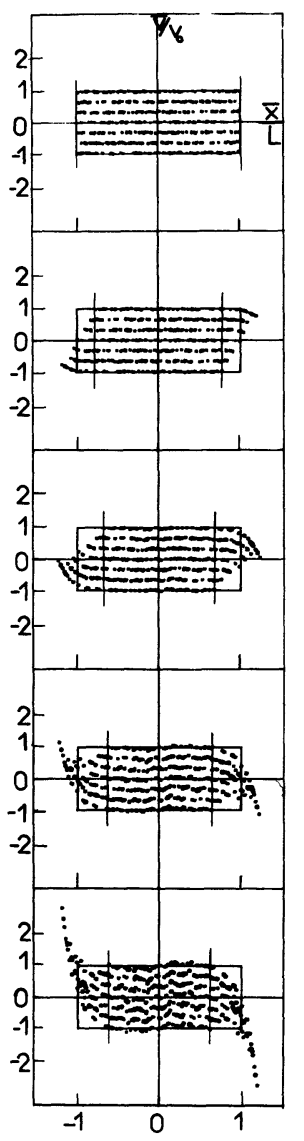

FIG. 1.

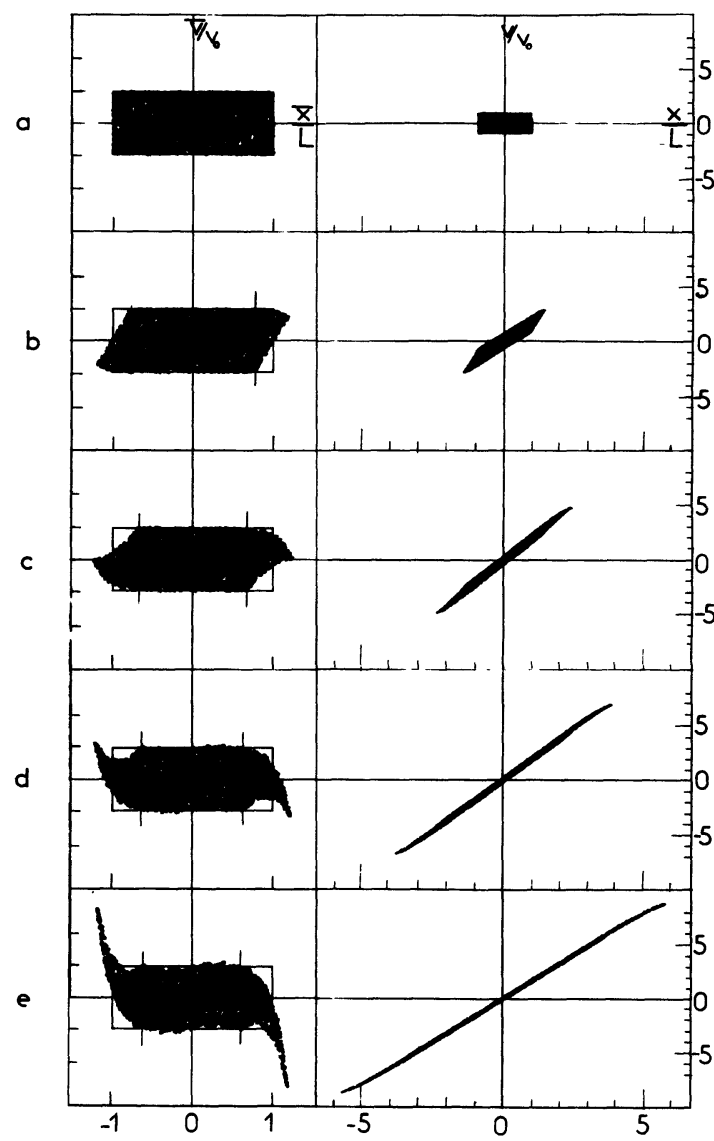

FIG. 2 .

Fig. 1. - Evolution dans les espaces de phases $x v$ (à droite) et $\overline{x v}$ (à gauche) d'un bâton horizontal du type water bag. A l'instant initial $\pm V_{0}$ sont les vitesses de coupures et le bâton a pour longueur $2 L$. Si $\Omega$ est la fréquence plasma correspondant à $A V_{0}=1 / 2$ densité à l'instant initial ( $A$ étant la valeur de $f$ à l'intérieur du water $b a g$ ) le problème est entièrement caractérisé par la valeur du paramètre $L \Omega / V_{0}$ qui ici vaut 2 . Les figures sont données respectivement pour $\Omega t=a) 0 ; b) 0,5 ; c) 1,0 ; d) 1,5 ; e) 2,0$. La zone non contaminée indiquée uniquement sur le diagramme $\overline{x v}$ est formée par le rectangle limité par les droites $\pm V_{0}$ et les deux traits verticaux dont l'évolution des abscisses est donnée par $\pm \bar{x}_{\mathrm{c}} / L=1-V_{0} / 2 \Omega L\left(\tau\left(1+\tau^{2}\right)+\right.$ Arc tg $\left.\tau\right)$. Dans le cas de figure le pourcentage de particules non contaminées tend pour $\tau \rightarrow \infty$ vers $1-\tau V_{0} / 4 \Omega L=0,6073$.

[Evolution in the phase spaces $x v$ (right) and $\overline{x v}$ (left) of an horizontal water bag rod. At $t=0$ the cutt off velocities are $\pm V_{0}$ and the rod length is $2 L$. We define $\Omega$ as the plasma frequency corresponding to the density $A V_{0}=1 / 2$ of the density at $t=0$. ( $A$ being the value of the phase space density inside the bag). The problem is entirely characterized by the value of the parameter $L \Omega / V_{0}$ (here equal to 2 ). Figures are respectively given for $\Omega t=a) 0 ; b$ ) $0.5 ; c) 1.0 ; d) 1.5 ; e) 2.0$. The uncontaminated zone is indicated only on the $\overline{x v}$ phase space and is a rectangle delineated by the straight lines $\pm V_{0}$ and the two vertical lines whose abscisses are given by

$$
\pm \bar{x}_{\mathrm{c}} / L=1-V_{0} / 2 \Omega L\left(\tau\left(1+\tau^{2}\right)+\operatorname{Arctg} \tau\right) .
$$

Here for $\tau \rightarrow \infty$ the percentage of uncontaminated particles go to $1-\tau V_{0} / 4 \Omega L=0.6073$.]

Fig. 2. - Même diagramme $\overline{x v}$ que pour la figure 1 avec indication de l'évolution de 7 groupes de particules dont les vitesses initiales sont $\pm V_{0}, \pm 2 V_{0} / 3, \pm V_{0} / 3$ et 0 . On voit que dans la zone non contaminée $\bar{v}$ est un invariant.

[Same diagram $\overline{x v}$ as in figure 1 with indication of the evolution of seven groups of particles with initial velocities $\pm V_{0}, \pm 2 V_{0} / 3, \pm V_{0} / 3$ and 0 . It can be seen that in the uncontaminated zone $\bar{v}$ is an invariant.]

la solution est

$$
x=\left(1+\alpha \cos \Omega_{\mathrm{p}} t\right)\left[\frac{\xi}{1+\alpha}+\eta\left(\frac{1+\alpha}{\Omega_{\mathrm{p}}}\right) I\left(\Omega_{\mathrm{p}} t\right)\right]
$$

dans (17) la fonction $I$ est donnée par

$$
\begin{aligned}
I(z)= & \int_{0}^{z} \frac{\mathrm{d} t^{\prime}}{\left(1+\alpha \cos t^{\prime}\right)^{2}} \\
= & \frac{1}{1-\alpha^{2}} \frac{-\alpha \sin z}{1+\alpha \cos z}+\frac{2}{\sqrt{1-\alpha^{2}}} \times \\
& \times \operatorname{Arctg} \frac{\sqrt{1-\alpha^{2} \operatorname{tg}(z / 2)}}{1+\alpha} .
\end{aligned}
$$


On calcule $u(x, t)$ donné par (3) et (10)

$$
u=\frac{-\alpha \Omega_{\mathrm{p}} \sin \Omega_{\mathrm{p}} t}{1+\alpha \cos \Omega_{\mathrm{p}} t} x
$$

et on obtient l'invariant

$$
\left[\frac{\mathrm{d} x}{\mathrm{~d} t}-u(x, t)\right] \frac{1+\alpha \cos \Omega_{\mathrm{p}} t}{1+\alpha}=\eta .
$$

Malheureusement la contamination du plasma est très différente de celle du faisceau. En particulier pour $\alpha \rightarrow 0$ on voit sur (17) que le mouvement de la particule est très voisin du mouvement balistique $x=\xi+\eta t$ et la contamination associée au point $x=L, \dot{x}=-V_{0}$ progresse vers le centre à cette vitesse $V_{0}$.

5. Conclusion. - Cette étude complète le travail exposé en [2] où nous étions partis des solutions self-similaires. Ces solutions étaient caractérisées par un exposant $\alpha$ et par $T$ le temps d'évolution. La famille $\alpha=-1 \Omega T=1$ (le bâton) est retrouvée en partant de l'hypothèse (1) et généralisée au cas d'une inclinaison quelconque pour un faisceau. De plus nous pouvons traiter le cas plasma, ce qui n'était pas possible à partir des équations self-similaires. Dans le cas faisceau la contamination peut n'être que partielle à cause de la nature explosive de la solution dans l'espace des phases et, par conséquent, d'un certain ordre qui finit par prévaloir. Rien de tel malheureusement n'est possible pour le cas plasma (ni d'ailleurs pour le cas gravitationnel) où le système oscille à la fréquence $\Omega_{\mathrm{p}}$. Pour les particules non contaminées un invariant absolu peut être obtenu.

Malgré les limitations sur le temps de validité qu'il faut parfois introduire, ces calculs ont le mérite de donner des solutions exactes du système VlasovPoisson pour des conditions initiales bien précises. Elles invitent à continuer l'étude des 'solutions selfsimilaires pour d'autres valeurs de $\alpha$ et de $T$ et également à regarder d'autres groupes de transformation comme les groupes de Lie [4].

\section{Bibliographie}

[1] Dawson, J. M., Phys. Rev. 113 (1959) 383.

[2] Burgan, J. R., Gutierrez, J., Fijalkow, E., Navet, M. and FEIX, M. R., Self similar solutions for Vlasov and Water Bag models. Submitted for publication to Journal of Plasma Physics.

[3] Navet, M. and Bertrand, P., Phys. Lett. 34A (1971) 117.

[4] AMES, W. F., Non linear partial differential equations in engineering Vol 2 (Academic Press New York) 1972. 